\title{
Factores intrínsecos y extrínsecos asociados con caídas en adultos mayores: estudio de casos y controles en México
}

\author{
Criselda Ríos-Fraustro, ${ }^{1}$ María E. Galván-Plata, ${ }^{2}$ Diana L. Gómez-Galicia, ${ }^{3}$ Liliana Giraldo-Rodríguez, ${ }^{4}$ \\ Marcela Agudelo-Botero ${ }^{5}$ y Dolores Mino-León ${ }^{6 *}$ \\ ${ }^{1}$ Facultad de Medicina, Universidad Nacional Autónoma de México, Ciudad de México; ${ }^{2}$ Coordinación de Investigación en Salud, Instituto Mexicano

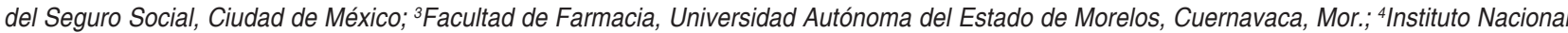 \\ de Geriatría, Secretaría de Salud, Ciudad de México; ${ }^{5}$ Facultad de Medicina, Centro de Investigación en Políticas, Población y Salud, Universidad \\ Nacional Autónoma de México, Ciudad de México; ${ }^{\circ}$ Unidad de Investigación en Epidemiología Clínica, Hospital de Especialidades, Centro Médico \\ Nacional Siglo XXI, Instituto Mexicano del Seguro Social, Ciudad de México. México
}

\section{Resumen}

Antecedentes: Las caídas tienen un origen multifactorial. Objetivo: Estimar el riesgo de caídas y su asociación con algunos factores intrínsecos y extrínsecos en adultos mayores. Métodos: Estudio de casos y controles que incluyó pacientes de ambos sexos con edades $\geq 60$ años. Los casos fueron pacientes que ingresaron al servicio de urgencias de un hospital de segundo nivel, con diagnóstico de lesión o fractura secundaria a una caída; los controles fueron pacientes que acudieron a unidades de medicina familiar. El análisis estadístico que se realizó fue descriptivo, bivariante y multivariante. Se utilizó el programa SPSS versión 22.0. Resultados: Se incluyeron 342 pacientes (171 casos y 171 controles). La edad promedio fue $76.1 \pm 8.8$ años, el 66 \% fueron mujeres y por autorreporte el 97.1 \% tenían enfermedades crónicas. Se observaron diferencias en el índice de masa corporal, en la proporción de casos con deterioro cognitivo, uso de dispositivos para caminar y dependencia para realizar actividades básicas e instrumentales de la vida diaria. El análisis multivariante ajustado reveló asociación entre el evento caída con deterioro cognitivo y dependencia para realizar actividades instrumentales de la vida diaria. Conclusiones: El deterioro cognitivo y la dependencia para realizar actividades instrumentales de la vida diaria se asociaron al riesgo de caer.

PALABRAS CLAVE: Caídas. Adulto mayor. Factores de riesgo. Adultos que viven en comunidad. México.

\section{Intrinsic and extrinsic factors associated with falls in older adults: a case-control study in Mexico}

\section{Abstract}

Background: Falls have a multifactorial origin. Objective: To estimate the risk of falls and their association with some intrinsic and extrinsic factors in elderly. Methods: Case-control study that included individuals of both genders aged $\geq 60$ years. Cases were patients who were admitted to the emergency department of a secondary care hospital diagnosed with injury or fracture secondary to a fall; the controls were patients who attended family medicine units. Descriptive, bivariate and multivariate statistical analysis was carried out. The SPSS program, version 22.0, was used. Results: Three-hundred and forty-two patients were included (171 cases and 171 controls). Mean age was $76.1 \pm 8.8$ years, $66 \%$ were women and $97.1 \%$ had self-reported chronic diseases. Differences were observed in body mass index, in the proportion of cases with cognitive impairment, use of walking devices and dependence to perform basic and instrumental activities of daily living. Adjusted multivariate analysis revealed an association between the fall event and cognitive impairment and dependence to perform instrumental

Correspondencia:

*Dolores Mino-León

E-mail: minod_mx@yahoo.com

DOI: 10.24875/GMM.20000111

0016-3813/C 2020 Academia Nacional de Medicina de México, A.C. Publicado por Permanyer. Este es un artículo open access bajo la licencia
Fecha de recepción: 10-03-2020

Fecha de aceptación: 26-05-2020
Gac Med Mex. 2021;157:133-139

Disponible en PubMed

www.gacetamedicademexico.com CC BY-NC-ND (http://creativecommons.org/licenses/by-nc-nd/4.0/). 
activities of daily living. Conclusions: Cognitive impairment and dependence to perform instrumental activities of daily living were associated with the risk of falling.

KEY WORDS: Falls. Older adult. Risk factors. Community-dwelling adults. Mexico.

\section{Antecedentes}

Diversos estudios poblacionales han descrito la epidemiología de las caídas en adultos mayores en diferentes contextos y en estos estudios se han reportado tasas menores (0.3-1.6 persona al año) en personas que viven en comunidad, quienes en general están sanas, que en personas institucionalizadas, en quienes las tasas son superiores (0.6-3.6 cama anual); además, se han descrito diferencias entre hombres y mujeres y aumento de las caídas a mayor edad..$^{1-3}$ Un estudio realizado en la región de las Américas encontró que la proporción de adultos mayores que sufrieron una caída al año osciló entre el $21.6 \%$ en Barbados y el $34 \%$ en Chile, seguido este último por México (33.5 \%). ${ }^{4}$ De acuerdo con la Organización Mundial de la Salud las caídas se definen como acontecimientos involuntarios que hacen perder el equilibrio y dar con el cuerpo en tierra u otra superficie firme que lo detenga..$^{5}$ Las caídas, así como las lesiones que estas ocasionan en los adultos mayores se consideran un problema mundial de salud pública, debido a su impacto sobre la frecuencia de fracturas, desarrollo del síndrome poscaída, hospitalización y mortalidad, entre otras complicaciones; en específico, el síndrome poscaída se ha asociado a confusión, inmovilización, depresión y pérdida de la autonomía. ${ }^{6-9}$ En la literatura se menciona que las caídas tienen un origen multifactorial ${ }^{6} \mathrm{y}$ algunos autores han propuesto clasificar los factores de riesgo en intrínsecos y extrínsecos. Los intrínsecos se relacionan con el estado funcional y de salud del individuo (comorbilidades, incapacidad funcional, alteraciones del equilibrio, movilidad y problemas para realizar actividades de la vida diaria) y los factores extrínsecos incluyen el uso de medicamentos (polimedicación, prescripción potencialmente inapropiada [PPI] y grupos terapéuticos específicos), uso de prótesis, dispositivos de ayuda para caminar y condiciones del entorno ambiental (iluminación deficiente y falta de equipo de seguridad en el baño, entre otros). ${ }^{10-13}$ El objetivo de este estudio fue estimar el riesgo de sufrir una caída asociado con algunos factores intrínsecos (condiciones clínicas y funcionales) y extrínsecos (medicamentos y uso de dispositivos de ayuda para caminar) en una muestra de adultos mayores que reciben atención médica en el Instituto Mexicano del Seguro Social (IMSS).

\section{Material y métodos}

\section{Diseño del estudio y muestra}

Se realizó un estudio de casos y controles, que incluyó pacientes de ambos sexos con edad $\geq 60$ años. Para el cálculo del tamaño de muestra se empleó un nivel de confianza al $95 \%$, un $80 \%$ de potencia y relación caso:control 1:1. El estudio se realizó de noviembre de 2014 a agosto de 2015.

\section{Definición de caso y control}

Se definió como caso a aquella persona que como consecuencia de un acontecimiento se precipitó desde su altura al suelo de forma involuntaria. Los casos se captaron de forma sistemática en el servicio de urgencias de un hospital general regional del IMSS de la Ciudad de México, con diagnóstico de lesión y/o fractura secundaria a caída. Se excluyeron los casos en los cuales la caída fue secundaria a un accidente de tránsito o intencionada; cuando por autorreporte o por información proporcionada por el familiar se conoció que el caso había tenido una o más caídas durante los seis meses previos al actual ingreso, o bien, que padeciera alguna de las siguientes enfermedades: vértigo postural, laberintitis, insuficiencia vertebrobasilar o que no tomara medicamentos de forma crónica. Los controles se recolectaron en la unidad de medicina familiar (UMF) a la que acudía el caso a recibir atención médica de primer contacto y se aparearon por edad y sexo; se excluyeron controles que en su expediente clínico se documentó que habían sufrido una caída durante los seis meses previos a la fecha de haber sido seleccionados. Todos los pacientes firmaron carta de consentimiento informado.

\section{Variables}

- Sociodemográficas: edad, sexo, escolaridad (básica $\leq 10$ años y superior $>10$ años), productivo en términos de trabajo y vivir solo. 
- Variables intrínsecas:

- Clínicas: índice de masa corporal (IMC), discapacidad visual (ceguera, miopía, astigmatismo, hipermetropía, glaucoma y cataratas); deterioro cognitivo (calificación $\leq 23$ en la prueba Mini-Mental): ${ }^{14}$ número de enfermedades crónicas y alcoholismo (consumo de al menos una vez a la semana).

- Funcionalidad: actividades básicas de la vida diaria (ABVD) evaluadas con el índice de Katz $^{15}$ y actividades instrumentales de la vida diaria (AIVD) con la Escala de Lawton y Brody. ${ }^{16}$

- Variables extrínsecas:

- Medicamentos: número de fármacos empleados para el tratamiento de enfermedades crónicas, polimedicación ( $\geq 5$ medicamentos), PPI de acuerdo con los criterios de Beers y de detección de prescripciones potencialmente inapropiadas en adultos mayores (por sus siglas en inglés STOPP); ${ }^{17,18}$ medicamentos que aumentan el riesgo de caídas (por sus siglas en inglés FRID), en los que incluimos: a) agentes cardiovasculares (metoprolol, propranolol, captopril, enalapril, lisinopril, ramipril, amlodipino, felodipino, nifedipino, verapamilo, prazosina, hidralazina, hidroclorotiazida y furosemida); b) fármacos con efecto anticolinérgico (bromuro de ipratropio, bromuro de tiotropio, oxibutinina, atropina, imipramina, amitriptilina y difenhidramina); c) neurolépticos/antipsicóticos (levomepromazina, trifluoperazina, haloperidol, flupentixol, ziprasidona, clozapina, olanzapina, quetiapina, risperidona), y d) hipnóticos y sedantes (alprazolam, lorazepam, bromazepam y diazepam).

- Uso de dispositivos para caminar (bastón, andadera, muletas y silla de ruedas).

\section{Recogida de datos}

Diariamente, uno de los investigadores (RFC) revisaba las notas clínicas de los pacientes que habían ingresado al servicio urgencias para identificar los casos que cumplían con los criterios de inclusión; una vez que el caso aceptó participar en el estudio, se solicitó la firma de la carta de consentimiento informado y se aplicó el cuestionario inicial (datos sociodemográficos, historia clínica y farmacológica [seis meses previos a haber sufrido la caída], problemas visuales y consumo de alcohol), el cuestionario del índice de Katz, la escala de Lawton y Brody y la prueba Mini-Mental. Esta última evaluación se realizó si el estado de salud del paciente lo permitía, de no ser así se aplazó hasta que mejorara la condición clínica del paciente.

Para captar a los controles, dos investigadores (RFC, GGDL) acudían a la UMF a la que asistía el caso a recibir atención médica de primer contacto, para revisar los registros e identificar a un paciente del mismo sexo y edad del caso; una vez identificado el control, era localizado por llamada telefónica para invitarlo y se le otorgaba una cita en la UMF. Durante la cita se le pidió firma de la carta de consentimiento informado y se le aplicaron los instrumentos de recolección de datos y de escalas que se le aplicaron al caso.

\section{Análisis estadístico}

Para las variables continuas se empleó media \pm desviación estándar y frecuencias y porcentajes para las variables categóricas. Se aplicaron pruebas de contrastación (Chi-cuadrada de Pearson y $\mathrm{t}$ de Student para muestras independientes). Se utilizaron modelos de regresión logística (simples y ajustados por variables sociodemográficas) para estimar el riesgo de caer asociado a factores intrínsecos y extrínsecos (razones de momios [OR] e intervalos de confianza [IC] al $95 \%$ ). El nivel de significancia estadística que se empleó en todas las pruebas fue de $p<0.05$. Se utilizó el paquete estadístico SPSS versus 22.0 (SPSS Inc., Chicago, IL) para Windows.

\section{Resultados}

Se incluyeron 342 adultos (171 casos y 172 controles) $\geq 60$ años. La edad promedio fue 76.1 años \pm 8.8 años; el $66 \%$ fueron mujeres y el $97.1 \%$ por autorreporte padecían al menos una enfermedad crónica, las más frecuentes fueron hipertensión arterial (67\%), diabetes mellitus tipo 2 (36\%), gastritis (24\%), dislipidemia (13\%) y enfermedad articular degenerativa (11\%). Los fármacos referidos con mayor frecuencia de consumo fueron vitamina B12 (B03BA), antihipertensivos (C09CA01 y C09AA02), hipoglucemiantes (A10BA02) y antiinflamatorios y analgésicos (N02BA01 y N02BE01).

No se observaron diferencias en el análisis bivariado (casos versus controles) en escolaridad, tener actividad laboral y vivir solo. Respecto a las variables clínicas se observó que el promedio del IMC fue menor 
Tabla 1. Características de los controles y los casos

\begin{tabular}{|c|c|c|c|}
\hline Variables & $\begin{array}{c}\text { Controles } \\
n=171 \\
n(\%)\end{array}$ & $\begin{array}{l}\text { Casos } \\
n=171 \\
n(\%)\end{array}$ & $p$ \\
\hline $\begin{array}{l}\text { Sociodemográficas } \\
\text { Edad (años)* }\end{array}$ & $75.7 \pm 8.5$ & $76.4 \pm 9.0$ & 0.450 \\
\hline $\begin{array}{l}\text { Sexo } \\
\text { Mujer } \\
\text { Hombre }\end{array}$ & $\begin{array}{l}113(66.1) \\
58(33.9)\end{array}$ & $\begin{array}{c}113(66.1) \\
58(33.9)\end{array}$ & 1.000 \\
\hline $\begin{array}{l}\text { Educación } \\
\text { Básica } \leq 10 \text { años }\end{array}$ & $129(75.4)$ & $131(76.6)$ & 0.450 \\
\hline $\begin{array}{l}\text { Productivo en términos de } \\
\text { trabajo (sí) }\end{array}$ & $9(5.3)$ & $15(8.8)$ & 0.145 \\
\hline Vivir solo (sí) & $31(18.1)$ & 21 (12.3) & 0.087 \\
\hline $\begin{array}{l}\text { Clínicas } \\
\text { Índice de masa corporal } \\
\text { Discapacidad visual } \\
\text { Deterioro cognitivo }{ }^{\dagger} \\
\text { Número de enfermedades } \\
\text { crónicas } \\
\text { Alcoholismo (sí) }\end{array}$ & $\begin{array}{l}25.5 \pm 6.7 \\
141(82.5) \\
21(12.3) \\
2.7 \pm 1.4 \\
20(11.7)\end{array}$ & $\begin{array}{l}24.1 \pm 7.0 \\
149(87.1) \\
50(39.9) \\
2.9 \pm 1.6 \\
28(16.4)\end{array}$ & $\begin{array}{l}0.041 \\
0.146 \\
0.000 \\
0.077 \\
0.138\end{array}$ \\
\hline $\begin{array}{l}\text { Funcionalidad } \\
\text { Dependencia en ABVD } \\
\text { Dependencia en AIVD }\end{array}$ & $\begin{array}{l}19(11.1) \\
76(44.4)\end{array}$ & $\begin{array}{c}40(23.4) \\
135(78.9)\end{array}$ & $\begin{array}{l}0.002 \\
0.000\end{array}$ \\
\hline $\begin{array}{l}\text { Medicamentos } \\
\text { Número de fármacos* } \\
\text { Polimedicación } n^{\ddagger} \\
\text { PPI Beers } \\
\text { PPI STOPP } \\
\text { FRID }\end{array}$ & $\begin{array}{c}5 \pm 3 \\
91(53.2) \\
53(31.0) \\
67(39.2) \\
122(71.3)\end{array}$ & $\begin{array}{c}5 \pm 3 \\
94(55.0) \\
58(33.9) \\
74(43.3) \\
128(74.9)\end{array}$ & $\begin{array}{l}0.638 \\
0.828 \\
0.644 \\
0.510 \\
0.542\end{array}$ \\
\hline $\begin{array}{l}\text { Otra } \\
\text { Uso de dispositivos para } \\
\text { caminar }\end{array}$ & $47(27.5)$ & $69(40.4)$ & 0.008 \\
\hline \multicolumn{4}{|c|}{$\begin{array}{l}\text { ABVD }=\text { actividades básicas de la vida diaria; } A I V D=\text { Actividades instrumentales de } \\
\text { la vida diaria; } P P I=\text { prescripción potencialmente inapropiada; } S T O P P=\text { prescripciones } \\
\text { potencialmente inapropiadas en adultos mayores; } F \mathrm{FID}=\text { medicamentos que aumentan } \\
\text { el riesgo de caídas. } \\
{ }^{\text {PPromedio }} \pm \text { desviación estándar. } \\
{ }^{+} \text {Mini-Mental } \leq 23 \text {. } \\
\$ \geq 5 \text { medicamentos. }\end{array}$} \\
\hline
\end{tabular}

en los casos que en los controles y una mayor proporción de casos tenían deterioro cognitivo $(p<0.05)$. Con respecto a la funcionalidad, una mayor proporción de casos tenían dependencia para realizar ABVD y AIVD y estas diferencias fueron significativas. No se observaron diferencias significativas en el número de fármacos, en las proporciones de polimedicación, de PPI por criterios de Beers y STOPP y FRID. Por último, la proporción de casos que usaban dispositivos para caminar fue más alta en los casos que en los controles $(p=0.008)$ (Tabla 1).

Los OR mostraron que el IMC, el deterioro cognitivo, la dependencia funcional (ABVD y AIVD) y usar
Tabla 2. Modelo de regresión simple

\begin{tabular}{|c|c|c|}
\hline Factor de riesgo & OR (IC $95 \%)$ & p \\
\hline $\begin{array}{l}\text { Educación (referencia: > } 10 \text { años) } \\
\leq 10 \text { años }\end{array}$ & $1.066(0.649-1.752)$ & 0.800 \\
\hline $\begin{array}{l}\text { Productivo en términos de } \\
\text { trabajo (referencia: si) } \\
\text { No }\end{array}$ & $0.578(0.246-1.359)$ & 0.209 \\
\hline $\begin{array}{l}\text { Vivir solo (referencia: no) } \\
\text { Sí }\end{array}$ & $0.632(0.347-1.152)$ & 0.134 \\
\hline Índice de masa corporal & $0.967(0.936-0.999)$ & 0.044 \\
\hline $\begin{array}{l}\text { Discapacidad visual (referencia: no) } \\
\text { Sí }\end{array}$ & $1.441(0.794-2.616)$ & 0.230 \\
\hline $\begin{array}{l}\text { Deterioro cognitivo* (referencia: no) } \\
\text { Sí }\end{array}$ & $3.189(1.812-5.613)$ & 0.000 \\
\hline Número de enfermedades crónicas & $1.132(0.986-1.300)$ & 0.078 \\
\hline $\begin{array}{l}\text { Alcoholismo (referencia: no) } \\
\text { Sí }\end{array}$ & $1.478(0.797-2.742)$ & 0.215 \\
\hline $\begin{array}{l}\text { Dependencia en ABVD } \\
\text { (referencia: no) } \\
\text { Sí }\end{array}$ & $2.443(1.349-4.424)$ & 0.003 \\
\hline $\begin{array}{l}\text { Dependencia en AIVD } \\
\text { (referencia: no) } \\
\text { Sí }\end{array}$ & $4.668(2.913-7.542)$ & 0.000 \\
\hline $\begin{array}{l}\text { Polimedicación }{ }^{\dagger} \text { (referencia: no) } \\
\text { Sí }\end{array}$ & $1.073(0.701-1.642)$ & 0.745 \\
\hline $\begin{array}{l}\text { PPI Beers (referencia: no) } \\
\text { Sí }\end{array}$ & $1.143(0.726-1.798)$ & 0.564 \\
\hline $\begin{array}{l}\text { PPI STOPP (referencia: no) } \\
\text { Sí }\end{array}$ & $1.184(0.770-1.822)$ & 0.442 \\
\hline $\begin{array}{l}\text { FRID (referencia: no) } \\
\text { Sí }\end{array}$ & $1.196(0.741-1.930)$ & 0.465 \\
\hline $\begin{array}{l}\text { Uso de dispositivos para caminar } \\
\text { (referencia: no) } \\
\text { Sí }\end{array}$ & $1.785(1.134-2.810)$ & 0.012 \\
\hline $\begin{array}{l}\text { OR = razón de momios; IC = intervalo de confiar } \\
\text { la vida diaria; } A \text { AVD = Actividades instrumentales } \\
\text { potencialmente inapropiada; } \\
\text { FRID = medicamentos que aumentan el riesgo c } \\
{ }^{*} \text { Mini-Mental } \leq 23 \text {. } \\
\geq \leq 5 \text { medicamentos. }\end{array}$ & $\begin{array}{l}\text {; } A B V D=\text { actividades bás } \\
\text { e la vida diaria; } P P I=\text { pres } \\
\text { caídas. }\end{array}$ & \\
\hline
\end{tabular}

dispositivos para caminar fueron variables que se asociaron al riesgo de caer (Tabla 2). Por último, el modelo multivariado ajustado mostró que el deterioro cognitivo y dependencia para realizar AIVD fueron variables que mantuvieron asociación significativa con riesgo de caer (Tabla 3).

\section{Discusión}

En la literatura se menciona que las caídas tienen un origen multifactorial y se ha propuesto clasificar los 
Tabla 3. Modelo de regresión logística múltiple ajustado por variables sociodemográficas

\begin{tabular}{|c|c|c|}
\hline Factor de riesgo & OR (IC $95 \%)$ & p \\
\hline Educación $\leq 10$ años & $0.788(0.447-1.386)$ & 0.408 \\
\hline Productivo en términos de trabajo & $0.542(0.208-1.414)$ & 0.211 \\
\hline Vivir solo & $0.878(0.440-1.750)$ & 0.711 \\
\hline Índice de masa corporal & $0.967(0.932-1.002)$ & 0.064 \\
\hline Deterioro cognitivo* & $2.071(1.106-3.877)$ & 0.023 \\
\hline Dependencia en ABVD & $1.067(0.519-2.197)$ & 0.859 \\
\hline Dependencia en AIVD & $3.786(2.216-6.469)$ & 0.000 \\
\hline Uso de dispositivos para caminar & $0.974(0.556-1.706)$ & 0.926 \\
\hline
\end{tabular}

factores de riesgo asociados con este evento en intrínsecos y extrínsecos. ${ }^{10,19}$ En este estudio los factores intrínsecos (deterioro cognitivo y dependencia para realizar AIVD) fueron las variables que se asociaron con el riesgo caer; otros estudios que analizaron poblaciones diferentes han reportado resultados similares. ${ }^{20-22}$ Además, se ha documentado que personas con problemas del equilibrio y dificultad para realizar funciones ejecutivas (FE), tienen más riesgo de caer. ${ }^{20}$ Otro estudio reportó asociación de deterioro cognitivo leve (basado principalmente en alteraciones de las $\mathrm{FE}$ ) con aumento en el riesgo de $\operatorname{caer}^{21}$, y los resultados de un estudio longitudinal que incluyó a México detectaron asociación entre deterioro cognitivo y riesgo de caer. ${ }^{23}$ Por otro lado, se ha reportado que personas con demencia experimentan mayor inestabilidad de la marcha al ser contrastados con personas sin demencia, lo que se ha atribuido a la presencia de alteraciones a nivel cortical y específicamente deterioro de las $\mathrm{FE} .{ }^{24-27}$ Las $\mathrm{FE}$ son una serie de procesos cognitivos superiores que usan y modifican información de diversos sistemas sensoriales a nivel cortical, localizados en las regiones anterior y posterior del cerebro. ${ }^{28,29}$ Estas funciones integrativas son necesarias para realizar de forma eficaz acciones dirigidas a lograr un objetivo y mantener la atención. Por lo que se ha sugerido que son la base para que un individuo sea capaz de realizar de forma independiente las actividades de la vida diaria; ${ }^{30,31}$ y su declive afecta la capacidad de caminar de forma eficiente y segura. $28,29,32$

Algunas limitaciones del estudio son la falta de análisis de las características de la marcha y de problemas vestibulares e información de la caída, además de un número limitado de controles. Sin embargo, el estudio tiene fortalezas, ya que se evitó el sesgo del recuerdo al incluir casos incidentes, los controles fueron apareados con los casos respecto a variables biológicas (edad y sexo) y se extrajeron de la misma población de la que proceden los casos, por lo que tuvieron la misma oportunidad de haber estado expuestos a los mismos factores de riesgo. Otra fortaleza del estudio es que se exploró el impacto de los medicamentos (polimedicación, PPI por criterios de Beers y STOPP y FRID) sobre las caídas, lo que es importante, ya que estudios previos realizados en México no lo han analizado. ${ }^{33,34} \mathrm{Y}$ si consideramos que el patrón de consumo de medicamentos puede ser diferente entre países, el análisis de los medicamentos cobra relevancia; por ejemplo, se ha descrito similitud en el patrón de consumo entre México y Brasil $^{35}$, no así con países europeos, en donde los hipnóticos, sedantes y ansiolíticos son los medicamentos que con mayor frecuencia se reportan como PPI, mientras que en México son la prescripción de antiinflamatorios no esteroideos y glibenclamida. ${ }^{36}$

Los resultados de este estudio revelan un desafío importante sobre el cual el IMSS debe actuar y se relaciona con la necesidad de implementar en el primer nivel de atención la evaluación geriátrica sistemática, ya que el IMSS es la institución que brinda servicios médicos al $40.6 \%$ de los adultos mayores (edad $\geq 60$ años) del país. ${ }^{37}$ Esta acción permitiría identificar a la población en riesgo ${ }^{38}$ que podría beneficiarse de acciones oportunas que han demostrado un impacto positivo sobre la cognición ${ }^{39}$ y a su vez disminuir el riesgo de caídas.

\section{Conclusiones}

Los resultados del estudio indican que los adultos mayores con deterioro cognitivo y dependencia en las AIVD tienen mayor riesgo de caer, lo cual es consistente con la relación descrita entre el déficit de las FE y las caídas y estos resultados abren la posibilidad de detectar alteraciones tempranas de las FE e iniciar tratamiento para mejorarlas y, en consecuencia, reducir el riesgo de caer.

\section{Agradecimientos}

A las autoridades de la Delegación Sur de la Ciudad de México, del IMSS; al director del Hospital General Regional N. ${ }^{\circ}$ 2, Dr. Miguel Chávez 
Covarrubias; al coordinador clínico de Educación e Investigación del HGR N. 2. A los médicos Guillermo Alejandro Salas Morales y Luis Alberto Guizar García, del Servicio de Medicina Interna del HGR N. ${ }^{\circ} 2$, así como a las autoridades de las UMF números 7,10 , 15, 19, 43 y 46, por su apoyo para llevar a cabo esta investigación. Al profesor Albert Figueras Suñé, por sus sugerencias.

\section{Conflicto de intereses}

Los autores declaran no tener conflicto de intereses alguno.

\section{Financiamiento}

Instituto Nacional de Geriatría, Secretaría de Salud, México.

\section{Responsabilidades éticas}

Protección de personas y animales. Los autores declaran que para esta investigación no se han realizado experimentos en seres humanos ni en animales.

Confidencialidad de los datos. Los autores declaran que han seguido los protocolos de su centro de trabajo sobre la publicación de datos de pacientes.

Derecho a la privacidad y consentimiento informado. Los autores han obtenido el consentimiento informado de los pacientes y/o sujetos referidos en el artículo. Este documento obra en poder del autor de correspondencia.

\section{Bibliografía}

1. Rubenstein LZ. Falls in older people: epidemiology, risk factors and strategies for prevention. Age Ageing. 2006;35(Suppl 2):ii37-ii41.

2. Peel NM, Kassulke DJ, McClure RJ. Population based study of hospitalised fall related injuries in older people. Inj Prev. 2002;8:280-3

3. Stalenhoef PA, Diederiks JP, Knottnerus JA, Kester AD, Crebolder HF A risk model for the prediction of recurrent falls in community-dwelling elderly: a prospective cohort study. J Clin Epidemiol. 2002;55:1088-94.

4. Reyes-Ortiz CA, Al Snih S, Markides KS. Falls among elderly persons in Latin America and the Caribbean and among elderly Mexican-Americans. Rev Panam Salud Publica. 2005;17:362-9.

5. Caídas [Internet]. World Health Organization; 16 de enero de 2018 [citado: 30 de mayo de 2018]. Disponible en: http://www.who.int/es/ news-room/fact-sheets/detail/falls

6. WHO Global Report on Falls Prevention in Older Age [Internet]. Ginebra, Suiza: World Health Organization; 2007. Disponible en: https://extranet. who.int/agefriendlyworld/wp-content/uploads/2014/06/WHo-Global-reporton-falls-prevention-in-older-age.pdf

7. Espino DV, Palmer RF, Miles TP, Mouton CP, Wood RC, Bayne NS, et al. Prevalence, incidence, and risk factors associated with hip fractures in community-dwelling older Mexican Americans: results of the Hispanic EPESE study. Establish Population for the Epidemiologic Study for the Elderly. J Am Geriatr Soc. 2000;48:1252-60.

8. Kannus P, Niemi S, Palvanen M, Parkkari J. Fall-induced injuries among elderly people. Lancet. 1997;350:1174.
9. Kempen GI, van Haastregt JC, McKee KJ, Delbaere K, Zijlstra GA. Socio-demographic, health-related and psychosocial correlates of fear of falling and avoidance of activity in community-living older persons who avoid activity due to fear of falling. BMC Public Health. 2009;9:170.

10. Huang AR, Mallet L, Rochefort CM, Eguale T, Buckeridge DL, Tamblyn R. Medication-related falls in the elderly: causative factors and preventive strategies. Drugs Aging. 2012;29:359-76.

11. Tinetti ME, Baker DI, McAvay G, Claus EB, Garrett P, Gottschalk M, et al. A multifactorial intervention to reduce the risk of falling among elderly people living in the community. N Engl J Med. 1994;331:821-7.

12. Hosseini $\mathrm{H}$, Hosseini N. Epidemiology and prevention of fall injuries among the elderly. Hosp Top. 2008;86:15-20.

13. Guelich MM. Prevention of falls in the elderly: a literature review. Top Geriatr Rehabil. 1999;15:15-25.

14. Folstein MF, Folstein SE, McHugh PR. "Mini-mental state". A practical method for grading the cognitive state of patients for the clinician. J Psychiatr Res. 1975;12:189-98.

15. Katz S, Ford AB, Moskowitz RW, Jackson BA, Jaffe MW. Studies of illness in the aged. The Index of Adl: A standardized measure of biological and psychosocial function. JAMA. 1963;185:914-9.

16. Lawton MP, Brody EM. Assessment of older people: self-maintaining and instrumental activities of daily living. Gerontologist. 1969:9:179-86.

17. American Geriatrics Society Beers Criteria Update Expert Panel. American Geriatrics Society 2015 Updated Beers Criteria for Potentially Inappropriate Medication Use in Older Adults. J Am Geriatr Soc. 2015;63:2227-46.

18. O'Mahony D, O'Sullivan D, Byrne S, O'Connor MN, Ryan C, Gallagher P. STOPP/START criteria for potentially inappropriate prescribing in older people: version 2. Age Ageing. 2015;44:213-8.

19. Santillana-Hernández SP, Alvarado-Moctezuma LE, Medina-Beltran GR, Gómez-Ortega G, Cortés-González RM. Caídas en el adulto mayor factores intrínsecos y extrínsecos. Rev Med Inst Mex Seguro Soc. 2002;40:489-93.

20. Delbaere K, Kochan NA, Close JC, Menant JC, Sturnieks DL, Brodaty H, et al. Mild cognitive impairment as a predictor of falls in community-dwelling older people. Am J Geriatr Psychiatry. 2012;20:845-53.

21. Delbaere K, Close JC, Heim J, Sachdev PS, Brodaty H, Slavin MJ, et al. A multifactorial approach to understanding fall risk in older people. J Am Geriatr Soc. 2010;58:1679-85.

22. Holtzer R, Friedman R, Lipton RB, Katz M, Xue X, Verghese J. The relationship between specific cognitive functions and falls in aging. Neuropsychology. 2007:2:540-8.

23. Stewart-Williams J, Kowal $P$, Hestekin $H$, O'Driscoll $T$, Peltzer $K$ Yawson A, et al. Prevalence, risk factors and disability associated with fall-related injury in older adults in low- and middle-incomecountries: results from the WHO Study on global AGEing and adult health (SAGE). BMC Med. 2015;13:147.

24. Allali G, Assal F, Kressig RW, Dubost V, Herrmann FR, Beauchet O. Impact of impaired executive function on gait stability. Dement Geriatr Cogn Disord. 2008:26:364-9.

25. Beauchet O, Kressig RW, Najafi B, Aminian K, Dubost V, Mourey F. Age-related decline of gait control under a dual-task condition. J Am Geriatr Soc. 2003:51:1187-8.

26. Verghese J, Lipton RB, Hall CB, Kuslansky G, Katz MJ, Buschke H. Abnormality of gait as a predictor of non-Alzheimer's dementia. N Engl J Med. 2002;347:1761-8.

27. Waite LM, Grayson DA, Piguet O, Creasey H, Bennett HP, Broe GA. Gait slowing as a predictor of incident dementia: 6-year longitudinal data from the Sydney Older Persons Study. J Neurol Sci. 2005;229-230:89-93.

28. Fuster JM. Frontal lobe and cognitive development. J Neurocytol. 2002;31:373-85.

29. Goethals I, Audenaert K, van de Wiele C, Dierckx R. The prefrontal cortex: insights from functional neuroimaging using cognitive activation tasks. Eur J Nucl Med Mol Imaging. 2004;31:408-16.

30. Romero-Ayuso DM. Actividades de la vida diaria. An Psicol. 2007;23: 264-71.

31. Reppermund S, Brodaty H, Crawford JD, Kochan NA, Draper B, Slavin MJ, et al. Impairment in instrumental activities of daily living with high cognitive demand is an early marker of mild cognitive impairment: the Sydney memory and ageing study. Psychol Med. 2013:43:2437-45.

32. Yogev-Seligmann G, Hausdorff JM, Giladi N. The role of executive function and attention in gait. Mov Disord. 2008;23:329-42; quiz 472.

33. Agudelo-Botero M, Giraldo-Rodríguez L, Murillo-González JC, Mino-León $D$, Cruz-Arenas E. Factors associated with occasional and recurrent falls in Mexican community-dwelling older people. PLoS One. 2018;13:e0192926.

34. Valderrama-Hinds LM, Al Snih S, Chen NW, Rodriguez MA, Wong R. Falls in Mexican older adults aged 60 years and older. Aging Clin Exp Res. 2018;30:1345-51

35. Oliveira MG, Amorim WW, de Jesus SR, Heine JM, Coqueiro HL,Passos LC. A comparison of the Beers and STOPP criteria for identifying the use of potentially inappropriate medications among elderly patients in primary care. J Eval Clin Pract. 2015;21:320-5. 
36. Mino-León D, Sánchez-García S, Giraldo-Rodríguez L, Reyes-Morales H. Potentially inappropriate prescribing to older adults in ambulatory care: prevalence and associated patient conditions. Eur Geriatr Med. 2019;10:639

37. Manrique-Espinoza B, Salinas-Rodríguez A, Moreno-Tamayo KM, Acosta-Castillo I, Sosa-Ortiz AL, Gutierrez-Robledo LM, et al. Condiciones de salud y estado funcional de los adultos mayores en México. Salud Publica Mex. 2013;55(Suppl 2):S323-31.
38. Mirelman A, Herman T, Brozgol M, Dorfman M, Sprecher E, Schweiger $A$, et al. Executive function and falls in older adults: new findings from a five-year prospective study link fall risk to cognition. PLoS One. 2012;7:e40297.

39. Colcombe SJ, Kramer AF, McAuley E, Erickson KI, Scalf P. Neurocognitive aging and cardiovascular fitness: recent findings and future directions. J Mol Neurosci. 2004;24:9-14. 